\title{
Low Rank Vector Bundles on the Grassmannian $G(1,4)$
}

\author{
Francesco Malaspina \\ Politecnico di Torino \\ Corso Duca degli Abruzzi 24, 10129 Torino, Italy \\ e-mail: malaspina@calvino.polito.it
}

September 25, 2018

\begin{abstract}
Here we define the concept of $L$-regularity for coherent sheaves on the Grassmannian $G(1,4)$ as a generalization of Castelnuovo-Mumford regularity on $\mathbf{P}^{\mathbf{n}}$. In this setting we prove analogs of some classical properties. We use our notion of $L$-regularity in order to prove a splitting criterion for rank 2 vector bundles with only a finite number of vanishing conditions. In the second part we give the classification of rank 2 and rank 3 vector bundles without "inner" cohomology (i.e. $H_{*}^{i}(E)=H^{i}(E \otimes \mathcal{Q})=0$ for any $i=2,3,4)$ on $G(1,4)$ by studying the associated monads.
\end{abstract}

\section{Introduction}

In chapter 14 of [11] Mumford introduced the concept of regularity for a coherent sheaf on a projective space $\mathbf{P}^{\mathbf{n}}$. It was soon clear that Mumford's definition of Castelnuovo-Mumford regularity was a key notion and a fundamental tool in many areas of algebraic geometry and commutative algebra. It has shown a very powerful tool, especially to study vector bundles. Chipalkatti generalized this notion to coherent sheaves on Grassmannians ([5]) and Costa and Miró-Roig gave on any $n$-dimensional smooth projective varieties with an $n$-block collection ([6]). In [2], it is introduced a simpler notion of regularity (called $G$-regularity) just on Grassmannians of lines by using the generalization of the Koszul exact sequence. It is a good tool because it includes some vector bundles which are not regular in the sense of [5] and can be use in order to characterize direct sums of line bundles and give a cohomological characterization of exterior and symmetric powers of the universal bundles of the Grassmannian. Unfortunately this notion, consists of infinitely many cohomological vanishings. However on $G(1,2)$ and $G(1,3)$ there are notions of regularity (which implies the $G$-regularity) with finite conditions: the Castelnuovo-Mumford regularity on $G(1,2) \cong \mathbf{P}^{2}$ and the Qregularity on $G(1,3) \cong \mathcal{Q}_{4}$ (see $\left.[3]\right)$.

In this paper we consider $G(1,4)$ and we give a notion of regularity with only a finite number of vanishing conditions. Next we show that the $L$-regularity implies the $G$-regularity and we prove the analogs of the classical properties on $\mathbf{P}^{\mathbf{n}+\mathbf{1}}$.

A well-known result of Horrocks (see [7]) characterizes the vector bundles without intermediate cohomology on a projective space as direct sum of line bundles. This criterion fails on

Mathematics Subject Classification 2000: 14F05, 14J60.

keywords: Universal Bundles on Grassmannians; Castelnuovo-Mumford regularity; Monads. 
more general varieties. There exist non-split vector bundles without intermediate cohomology. These bundles are called ACM bundles. For instance the universal bundles of a Grassmannian are ACM. Ottaviani generalized Horrocks criterion to quadrics and Grassmannians by giving cohomological splitting conditions for vector bundles (see [12, 13]). Arrondo and Graña in [1] gave a cohomological characterization of the universal bundles and a classification of ACM bundles on $G(1,4)$. In [2] Arrondo and the author generalized the first part of [1] by giving a cohomological characterization of exterior and symmetric powers of the universal bundles on any grassmannian of lines.

Here we apply our notion of regularity in order to prove a splitting criterion for rank 2 vector bundle (see Proposition 1.6). We require the vanishing of the intermediate cohomology only for some particular twist. So we have the analogous of [4] Corollary 1.8. on $\mathbf{P}^{n}$ and [3] Proposition 4.6. on $\mathcal{Q}_{n}$.

In the second part of the paper we deal with monads. A monad on $\mathbf{P}^{\mathbf{n}}$ or, more generally, on a projective variety $X$, is a complex of three vector bundles

$$
0 \rightarrow A \stackrel{\alpha}{\rightarrow} B \stackrel{\beta}{\rightarrow} C \rightarrow 0
$$

such that $\alpha$ is injective as a map of vector bundles and $\beta$ is surjective. Monads have been studied by Horrocks, who proved (see [7]) that every vector bundle on $\mathbf{P}^{\mathbf{n}}$ is the homology of a suitable minimal monad. This correspondence holds also on a projective variety $X$ $(\operatorname{dim} X \geq 3)$ if we fix a very ample line bundle $\mathcal{O}_{X}(1)$ (see 9$]$ ).

Rao, Mohan Kumar and Peterson on $\mathbf{P}^{\mathbf{n}}$ (see [8]), and the author on quadrics (see [9, 10]) gave a classification of rank 2 and 3 vector bundles without inner cohomology (i.e. $H_{*}^{1}(E)=$ $\left.\ldots=H_{*}^{n-1}(E)=0\right)$ by studying the associated minimal monads.

On $G(1,4)$ we say that a vector bundle is without inner cohomology if $H_{*}^{i}(E)=H^{i}(E \otimes \mathcal{Q})=0$ for any $i=2,3,4$. Then we classify the rank 2 and 3 vector bundles without inner cohomology. In particular we prove that there are no minimal monads with $A \neq 0$ or $C \neq 0$ associated to a rank 2 and 3 vector bundle without inner cohomology.

We are grateful to E. Arrondo for the useful discussions and his comments.

\section{Regularity on $G(1,4)$}

Throughout the paper $\mathbf{P}^{n}$ will denote the projective space consisting of the one-dimensional quotients of the $(n+1)$-dimensional vector space $V$ over an algebraically closed field $\mathbb{K}$ with characteristic zero. $G(1,4)$ (frequently denoted just by $G$ ) will be the Grassmann variety of lines in $\mathbf{P}^{\mathbf{4}}$. We consider the universal exact sequence on $G=G(1,4)$ :

$$
0 \rightarrow S^{\vee} \rightarrow V \otimes \mathcal{O}_{G} \rightarrow Q \rightarrow 0
$$

defining the universal bundles $S$ and $Q$ over $G$ of respective ranks 3 and 2. We will also write $\mathcal{O}_{G}(1)=\bigwedge^{2} Q \cong \bigwedge^{3} S$. In particular, we have natural isomorphisms

$$
S^{j} Q^{\vee} \cong\left(S^{j} Q\right)(-j)
$$

(where $S^{j}$ denotes the $j$-th symmetric power) and

$$
\bigwedge^{j} S^{\vee} \cong \bigwedge^{3-j} S(-1)
$$


The second exterior product in the left map of (1) is

$$
0 \rightarrow \bigwedge^{2} S^{\vee} \rightarrow \bigwedge^{2} V \otimes \mathcal{O}_{G} \rightarrow V \otimes Q \rightarrow S^{2} Q \rightarrow 0 .
$$

Observe now that we can glue the dual of (11) twisted by $\mathcal{O}_{G}(-1)$ with (4) and we obtain

$$
0 \rightarrow \mathcal{Q}(-2) \rightarrow V^{*} \otimes \mathcal{O}_{G}(-1) \rightarrow \bigwedge^{2} V \otimes \mathcal{O}_{G} \rightarrow V \otimes Q \rightarrow S^{2} Q \rightarrow 0 .
$$

Let us consider also the dual sequence twisted by $\mathcal{O}_{G}(-3)$ :

$$
0 \rightarrow S^{2} Q(-3) \rightarrow V^{*} \otimes Q(-2) \rightarrow \bigwedge^{2} V^{*} \otimes \mathcal{O}_{G}(-1) \rightarrow V \otimes \mathcal{O}_{G} \rightarrow Q \rightarrow 0 .
$$

If we glue (5) with (11) twisted by $\mathcal{O}_{G}(-2)$ we obtain

$$
\begin{gathered}
0 \rightarrow S^{\vee}(-2) \rightarrow V \otimes \mathcal{O}_{G}(-2) \rightarrow V^{*} \otimes \mathcal{O}_{G}(-1) \rightarrow \\
\rightarrow \bigwedge^{2} V \otimes \mathcal{O}_{G} \rightarrow V \otimes Q \rightarrow S^{2} Q \rightarrow 0 .
\end{gathered}
$$

We can also glue the dual of (4) twisted by $\mathcal{O}_{G}(-3)$ with (4) and we obtain

$$
\begin{gathered}
0 \rightarrow \bigwedge^{2} S^{\vee}(-3) \rightarrow \bigwedge^{2} V \otimes \mathcal{O}_{G}(-3) \rightarrow V \otimes Q(-3) \rightarrow \\
\rightarrow V^{*} \otimes Q(-2) \rightarrow \bigwedge^{2} V^{*} \otimes \mathcal{O}_{G}(-1) \rightarrow V \otimes \mathcal{O}_{G} \rightarrow Q \rightarrow 0 .
\end{gathered}
$$

Let us consider also the dual sequence twisted by $\mathcal{O}_{G}(-4)$ :

$$
\begin{gathered}
0 \rightarrow Q(-5) \rightarrow V^{*} \otimes \mathcal{O}_{G}(-4) \rightarrow \bigwedge^{2} V \otimes \mathcal{O}_{G}(-3) \rightarrow \\
\rightarrow V \otimes Q(-3) \rightarrow V^{*} \otimes Q(-2) \rightarrow \bigwedge^{2} V \otimes \mathcal{O}_{G}(-1) \rightarrow S^{\vee} \rightarrow 0 .
\end{gathered}
$$

Finally the top exterior product in the left map of (10) (twisted by $\mathcal{O}_{G}(-3)$ ) glued with the dual, it is the analogous in $G$ of the long Koszul exact sequence in the projective space. We have

$$
\begin{aligned}
0 & \rightarrow \mathcal{O}_{G}(-4) \rightarrow \bigwedge^{3} V \otimes \mathcal{O}_{G G}(-3) \rightarrow \bigwedge^{2} V \otimes Q(-3) \rightarrow V \otimes S^{2} Q(-3) \rightarrow \\
& \rightarrow V^{*} \otimes S^{2} Q(2) \rightarrow \bigwedge^{2} V^{*} \otimes Q(-1) \rightarrow \bigwedge^{3} V^{*} \otimes \mathcal{O}_{G} \rightarrow \mathcal{O}_{G}(1) \rightarrow 0 .
\end{aligned}
$$

Remark 1.1. Let us notice that all the symmetric powers (except the last) that appear in sequence (1) have order smaller than 2. This is not true for the analog sequence when $n>4$. For this reason the author in convinced that these ideas cannot be extended on $G(1, n)$ with $n>4$.

We are ready to introduce our notion of regularity: 
Definition 1.2. We say that a coherent sheaf $F$ on $G(1,4)$ is $m$-L-regular if the following conditions hold:

$$
\begin{aligned}
i & H^{1}(F(m-1))=H^{2}(F(m-2))=H^{3}(F(m-3))=H^{4}(F(m-3))=H^{5}(F(m-3))= \\
& H^{5}(F(m-4))=H^{6}(F(m-4))=0 . \\
\text { ii } & H^{2}(F \otimes Q(m-2))=H^{3}(F \otimes Q(m-3))=H^{4}(F \otimes Q(m-3))=H^{4}(F \otimes Q(m-4))= \\
& H^{5}(F \otimes Q(m-4))=0 . \\
\text { iii } & H^{3}\left(F \otimes S^{2} Q(m-3)\right)=H^{4}\left(F \otimes S^{2} Q(m-4)\right)=H^{5}\left(F \otimes S^{2} Q(m-5)\right)=0 .
\end{aligned}
$$

We will say L-regular instead of 0-L-regular.

Proposition 1.3. Let $F$ be a L-regular coherent sheaf on $G=G(1,4)$. For any $k \geq 0$,

(a) $F(k)$ is L-regular.

(b) $F(k)$ is generated by its global sections.

Proof. First of all let us prove that

$$
H^{6}(F \otimes Q(-5))=H^{6}\left(F \otimes S^{2} Q(-6)\right)=0
$$

From the sequence (5), tensored by $F(-3)$ we have that

$$
H^{6}(F(-4))=H^{5}(F(-3))=H^{4}(F \otimes Q(-3))=H^{3}\left(F \otimes S^{2} Q(-3)\right)=0,
$$

implies $H^{6}(F \otimes Q(-5))=0$.

From (6) tensored by $F(-3)$ we have that

$$
H^{6}(F \otimes Q(-5))=H^{5}(F(-4))=H^{4}(F(-3))=H^{3}(F \otimes Q(-3))=0,
$$

implies $H^{6}\left(F \otimes S^{2} Q(-6)\right)=0$.

Now let us show that

$$
H^{1}(F)=H^{2}(F(-1))=H^{3}(F(-2))=H^{4}(F(-2))=H^{5}(F(-2))=H^{6}(F(-3))=0 .
$$

Let us consider the sequence (11) tensored by $F(-1)$, since

$$
\begin{gathered}
H^{7}(F(-5))=H^{6}(F(-4))=H^{5}(F \otimes Q(-4))=H^{4}\left(F \otimes S^{2} Q(-4)\right)= \\
=H^{3}\left(F \otimes S^{2} Q(-3)\right)=H^{2}(F \otimes Q(-2))=H^{1}(F(-1))=0,
\end{gathered}
$$

we obtain $H^{1}(F)=0$.

If we tensor (11) by $F(-2)$, since

$$
\begin{aligned}
& H^{7}(F(-6))=H^{6}(F \otimes Q(-5))=H^{5}\left(F \otimes S^{2} Q(-5)\right)= \\
= & H^{4}\left(F \otimes S^{2} Q(-4)\right)=H^{3}(F \otimes Q(-3))=H^{2}(F(-2))=0,
\end{aligned}
$$

we obtain $H^{2}(F(-1))=0$.

If we tensor (11) by $F(-3)$, since 


$$
H^{6}\left(F \otimes S^{2} Q(-6)\right)=H^{5}\left(F \otimes S^{2} Q(-5)\right)=H^{4}(F \otimes Q(-4))=H^{3}(F(-3))=0,
$$

we obtain $H^{3}(F(-2))=0$.

Moreover, since

$$
H^{6}\left(F \otimes S^{2} Q(-5)\right)=H^{5}(F \otimes Q(-4))=H^{4}(F(-3))=0,
$$

we obtain $H^{4}(F(-2))=0$.

Since

$$
H^{6}(F \otimes Q(-4))=H^{5}(F(-3))=0,
$$

we obtain $H^{5}(F(-2))=0$ and clearly $H^{6}(F(-3))=0$.

Next we want show that

$$
H^{1}(F \otimes Q)=H^{2}(F \otimes Q(-1))=H^{3}(F \otimes Q(-2))=H^{4}(F \otimes(-2))=H^{5}(F \otimes Q(-3))=0
$$

Let us consider the sequence (11) tensored by $F(-3)$, since

$$
H^{6}(F(-4))=H^{5}(F(-3))=0,
$$

we obtain $H^{5}(F \otimes Q(-3))=0$.

If we tensor (1) by $F(-2)$, since

$$
H^{6}(F \otimes Q(-4))=H^{5}(F(-3))=H^{4}(F(-2))=0,
$$

we obtain $H^{4}(F \otimes Q(-2))=0$.

Moreover, since

$$
H^{6}(F \otimes Q(-5))=H^{5}(F \otimes Q(-4))=H^{4}(F(-3))=H^{3}(F(-2))=0,
$$

we obtain $H^{3}(F \otimes Q(-2))=0$.

If we tensor (10) by $F(-1)$, since

$$
H^{6}(F(-4))=H^{5}(F \otimes Q(-4))=H^{4}(F \otimes Q(-3))=H^{3}(F(-2))=H^{1}(F(-1))=0,
$$

we obtain $H^{2}(F \otimes Q(-1))=0$.

Let us prove finally that

$H^{2}\left(F \otimes S^{2} Q(-1)\right)=H^{3}\left(F \otimes S^{2} Q(-2)\right)=H^{4}\left(F \otimes S^{2} Q(-3)\right)=H^{5}\left(F \otimes S^{2} Q(-4)\right)=0$.

Let us consider the sequence (11) tensored by $F(-4)$, since

$$
H^{6}(F(-4))=H^{5}(F \otimes Q(-4))=0,
$$


we obtain $H^{5}\left(F \otimes S^{2} Q(-4)\right)=0$.

Moreover, tensoring (1) by $F(-3)$, since

$$
H^{6}(F(-4))=H^{5}(F(-3))=H^{4}(F \otimes Q(-3))=0,
$$

we obtain $H^{4}\left(F \otimes S^{2} Q(-3)\right)=0$.

If we tensor (10) by $F(-2)$, since

$$
H^{6}(F(-4))=H^{5}(F(-3))=H^{4}(F(-2))=H^{3}(F \otimes Q(-2))=0,
$$

we obtain $H^{3}\left(F \otimes S^{2} Q(-2)\right)=0$.

(b) We need the following lemma:

Lemma 1.4. Let $F$ be a L-regular coherent sheaf on $G$. Then, it is $G$-regular.

Proof. We only need to show that, for any $k \geq 0$,

$$
H^{1}(F \otimes Q(k-1))=H^{2}\left(F \otimes S^{2} Q(k-2)\right)=0 .
$$

From the sequence (44) tensored by $F(-4)$ we see that $H^{6}\left(F \otimes \bigwedge^{2} S^{\vee}(-4)\right)=0$. In fact

$$
H^{6}(F(-4))=H^{5}(F \otimes Q(-4))=H^{4}\left(F \otimes S^{2} Q(-4)\right)=0 .
$$

Let us tensorize the sequence (1) by $F(-1)$. Since

$$
\begin{gathered}
H^{6}\left(F \otimes \bigwedge^{2} S^{\vee}(-4)\right)=H^{5}(F(-4))=H^{4}(F \otimes Q(-4))= \\
=H^{3}(F \otimes Q(-3))=H^{2}(F(-2))=H^{1}(F(-1))=0,
\end{gathered}
$$

we have $H^{1}(F \otimes Q(-1))=0$.

From the sequence (11) tensored by $F(-4)$ we see that $H^{6}\left(F \otimes S^{\vee}(-4)\right)=0$. In fact

$$
H^{6}(F(-4))=H^{5}(F \otimes Q(-4))=0 .
$$

Let us tensorize the sequence (1) by $F(-2)$. Since

$$
H^{6}\left(F \otimes S^{\vee}(-4)\right)=H^{5}(F(-4))=H^{4}(F(-3))=H^{3}(F(-2))=H^{2}(F \otimes Q(-2))=0,
$$

we have $H^{2}(F \otimes Q(-2))=0$.

Now, since $F(k)$ is $L$-regular for any $k \geq 0$, we have the claimed vanishing for any $k \geq 0$.

Since $F$ is $G$-regular then by [2] Proposition 2.3. it is globally generated.

Definition 1.5. Let $F$ be a coherent sheaf on $G$. We define the $L$-regularity of $F$, Lreg $(F)$, as the least integer $m$ such that $F$ is $m$-L-regular. We set $\operatorname{Lreg}(F)=-\infty$ if there is no such an integer.

We can use the notion of $L$-regularity in order to prove a splitting criterion for rank 2 vector bundles on $G$ with only a finite number of vanishing conditions: 
Proposition 1.6. Let $E$ be a rank 2 bundle on $G$ with $\operatorname{Lreg}(E)=0$. Let us assume that

$$
H^{1}(E(-2))=H^{3}(E(-4))=H^{4}(E(-4))=H^{5}(E(-5))=0,
$$

and

$$
H^{2}(E \otimes Q(-3))=H^{3}(E \otimes Q(-4))=H^{4}(E \otimes Q(-5))=0 .
$$

Then $E \cong Q$ or $E \cong \mathcal{O} \oplus \mathcal{O}(a)$ with $a \geq 0$.

Proof. If we apply Le Potier vanishing theorem to a rank 2 bundle on $G$ with $\operatorname{Lreg}(E)=0$, we obtain $H^{i}(E(k-3))=0$ for any $i \geq 2$ and any $k \geq 0$, so we have $H^{2}(E(-3)=0$.

Since $\operatorname{Lreg}(E)=0, E$ is $L$-regular but $E(-1)$ not. $E(-1)$ is not $L$-regular if and only if one of the following conditions is satisfied:

$$
\begin{aligned}
& \text { i } H^{6}(E(-5)) \neq 0, \\
& \text { ii } H^{3}\left(E(-1) \otimes S^{2} Q(-3)\right) \neq 0, \\
& \text { iii } H^{5}(E(-1) \otimes Q(-4)) \neq 0, \\
& \text { iv } H^{4}\left(E(-1) \otimes S^{2} Q(-4)\right) \neq 0, \\
& \text { v } H^{5}\left(E(-1) \otimes S^{2} Q(-5)\right) \neq 0 .
\end{aligned}
$$

Let us consider one by one the conditions:

(i) Let $H^{6}(E(-5)) \neq 0$, so $H^{0}\left(E^{\vee}\right) \neq 0$ and $\mathcal{O}$ is a direct summand of $E$. Then $E \cong \mathcal{O} \oplus \mathcal{O}(a)$ with $a \geq 0$.

(ii) Let $H^{3}\left(E(-1) \otimes S^{2} Q(-3)\right) \neq 0$. Let us consider the exact sequence (6) tensored by $E(-1)$. Since

$$
H^{3}(E \otimes Q(-3))=H^{2}(E(-2))=H^{1}(E(-1))=0,
$$

we see that $H^{0}(E \otimes Q(-1)) \neq 0$.

From the sequence (5) tensored by $E(-4)$ we have that

$$
H^{6}(E(-5))=H^{5}(E(-4))=H^{4}(E \otimes Q(-4))=0,
$$

implies $H^{6}(E \otimes Q(-6)) \cong H^{3}\left(E(-1) \otimes S^{2} Q(-3)\right)$. But $H^{6}(E \otimes Q(-6)) \cong H^{0}\left(E^{\vee} \otimes Q\right)$.

Let us consider the following commutative diagram of natural morphisms:

$$
\begin{aligned}
& H^{3}\left(E \otimes S^{2} Q(-4)\right) \otimes H^{3}\left(E^{\vee} \otimes S^{2} Q(-3)\right) \quad \stackrel{\sigma}{\rightarrow} \quad H^{6}\left(S^{2} Q \otimes S^{2} Q(-7)\right) \\
& \uparrow \quad \uparrow \\
& H^{0}(E \otimes Q(-1)) \otimes H^{3}\left(E^{\vee} \otimes S^{2} Q(-3)\right) \quad \stackrel{\mu}{\rightarrow} \quad H^{3}\left(Q \otimes S^{2} Q(-4)\right) \cong \mathbb{C} \\
& \uparrow \\
& H^{0}\left(E \otimes Q^{\vee}\right) \otimes H^{0}\left(E^{\vee} \otimes Q\right) \quad \stackrel{\tau}{\rightarrow} \quad H^{0}\left(Q \otimes Q^{\vee}\right) \cong \mathbb{C} \\
& \uparrow \cong \\
& \operatorname{Hom}(Q, E) \otimes \operatorname{Hom}(E, Q) \quad \stackrel{\gamma}{\rightarrow} \quad \operatorname{Hom}(Q, Q)
\end{aligned}
$$

The map $\sigma$ comes from Serre duality and it is not zero, the right vertical map are isomorphisms and the left vertical map are surjective so also the map $\tau$ is not zero. This map is naturally identified with the map $\gamma$ consisting just of the composition of homomorphisms. This means that the composition of the following maps

$$
Q \rightarrow E \rightarrow Q
$$


is not zero. Since the endomorphisms of $Q$ are multiplications by scalars, we can assume (after multiplying by a suitable scalar) that the above composition is the identity. Now we can conclude that $E \cong Q$.

Now we have to show that the conditions (iii), (iv) and $(v)$ are not possible.

(iii) Let $H^{5}(E(-1) \otimes Q(-4)) \neq 0$. Since

$$
H^{6}(E(-5))=H^{5}(E(-5))=0
$$

we have

$$
H^{5}(E(-1) \otimes Q(-4)) \cong H^{6}\left(E \otimes S^{\vee}(-5)\right) \cong H^{0}\left(E^{\vee} \otimes S\right),
$$

so $H^{0}\left(E^{\vee} \otimes S\right) \neq 0$.

On other hand let us tensorize the sequence (10) by $E$. Since

$$
H^{5}(E(-4))=H^{4}(E(-3))=H^{3}(E \otimes Q(-3))=H^{2}(E \otimes Q(-2))=H^{1}(E(-1))=0,
$$

we have $H^{0}\left(E \otimes S^{\vee}\right)=0$. So we can conclude that $S$ is a direct summand of $E$. But $S$ has rank 3 then we have a contradiction.

(vi) First of all we claim that $H^{1}(E \otimes Q(-2))=0$.

If $H^{1}(E \otimes Q(-2)) \neq 0$ in fact, by arguing as above, we can conclude that $S^{\vee}$ is a direct summand of $E(-1)$. But $S^{\vee}$ has rank 3 then we have a contradiction.

Let $H^{4}\left(E(-1) \otimes S^{2} Q(-4)\right) \neq 0$. Let us consider the exact sequence (6) tensored by $E(-2)$. Since

$$
H^{4}(E \otimes Q(-4))=H^{3}(E \otimes Q(-4))=H^{2}(E(-3))=H^{1}(E(-2))=0,
$$

we have that

$$
H^{4}\left(E(-1) \otimes S^{2} Q(-4)\right) \cong H^{1}(E \otimes Q(-2)) .
$$

$(v)$ Let us consider the exact sequence (6) tensored by $E(-3)$. Since

$$
H^{5}(E \otimes Q(-5))=H^{4}(E(-4))=H^{3}(E(-3))=H^{2}(E \otimes Q(-3))=0,
$$

we have that $H^{5}\left(E \otimes S^{2} Q(-6)\right)=0$.

Remark 1.7. We found the analogous of [4] Corollary 1.8. and [3] Proposition 4.6. on G.

\section{Rank 2 and rank 3 vector bundles without inner cohomology}

We introduce the following definition:

Definition 2.1. We will call bundle without inner cohomology a bundle $E$ on $G$ with

$$
H_{*}^{i}(E)=H_{*}^{i}(E \otimes Q)=0, \text { for any } i=2,3,4 .
$$

In this section we classify all the rank 2 and rank 3 bundles without inner cohomology. Now we introduce the following tool: the monads.

Let $\mathcal{E}$ be a vector bundle on $G$. There is the corresponding minimal monad

$$
0 \rightarrow A \stackrel{\alpha}{\rightarrow} B \stackrel{\beta}{\rightarrow} C \rightarrow 0
$$

where $A$ and $C$ are sums of line bundles and $B$ satisfies: 
1. $H_{*}^{1}(B)=H_{*}^{n-1}(B)=0$

2. $H_{*}^{i}(B)=H_{*}^{i}(E) \quad \forall i, 1<i<5$.

A monad will be called minimal if the maps $\alpha$ and $\beta$ are minimal: the surjective map $\beta$ is said minimal if no direct summand of $\mathcal{C}$ is the image of a line subbundle of $\mathcal{B}$.

An equivalent condition is that no generator of $B$ can be sent in a generator of $C$.

$\alpha$ is minimal if the surjective $\alpha^{\vee}$ is minimal as defined for $\beta$.

If $M$ is a finitely generated graded module over the homogeneous coordinate $\operatorname{ring}$ of $G, S_{G}$, we denote by $\beta_{i}(M)$ the total Betti numbers of $M$. We will mainly use $\beta_{0}(M)$ which give the number of minimal generators of $M$.

Recall that if

$$
M \rightarrow N \rightarrow 0
$$

is a surjection of finitely generated graded $S_{G}$-modules, then $\beta_{0}(M) \geq \beta_{0}(N)$. Furthermore, if the inequality is strict, it means that a set of minimal generators of $M$ can be chosen in such a way that one of generators in the set maps to zero.

Remark 2.2. By [10] Theorem 2.2. any minimal monad

$$
0 \rightarrow \mathcal{A} \stackrel{\alpha}{\rightarrow} \mathcal{B} \stackrel{\beta}{\rightarrow} \mathcal{C} \rightarrow 0
$$

such that $\mathcal{A}$ or $\mathcal{C}$ are not zero, for a rank $r(r \leq 3)$ bundle with $H_{*}^{2}(E)=H_{*}^{4}(E)=0$, must satisfy the following conditions:

1. $H_{*}^{1}\left(\wedge^{2} \mathcal{B}\right) \neq 0, \beta_{0}\left(H_{*}^{1}\left(\wedge^{2} \mathcal{B}\right)\right) \geq \beta_{0}\left(H_{*}^{0}\left(S_{2} \mathcal{C}\right)\right)$, if $\mathcal{C}$ is not zero.

2. $H_{*}^{1}\left(\wedge^{2} \mathcal{B}^{\vee}\right) \neq 0, \beta_{0}\left(H_{*}^{1}\left(\wedge^{2} \mathcal{B}^{\vee}\right)\right) \geq \beta_{0}\left(H_{*}^{0}\left(S_{2} \mathcal{A}^{\vee}\right)\right)$, if $\mathcal{A}$ is not zero.

3. $H_{*}^{2}\left(\wedge^{2} \mathcal{B}\right)=H_{*}^{2}\left(\wedge^{2} \mathcal{B}^{\vee}\right)=0$.

Remark 2.3. Here we list the only non-zero intermediate cohomology of the universal bundles when tensored with $Q$ and $S^{\vee}$ (see [1] Table 1.3):

$$
h^{1}\left(Q \otimes S^{\vee}\right)=h^{5}(S \otimes Q(-5))=h^{2}\left(S^{\vee} \otimes S^{\vee}\right)=1 .
$$

We are ready to prove the main result of this section:

Theorem 2.4. On $G$ the only rank $r(r \leq 3)$ bundles without inner cohomology are (up to twist) the following:

1. for $r=2, Q$ and the sums of line bundles,

2. for $r=3, Q \oplus \mathcal{O}(a), S, S^{\vee}$ and the sums of line bundles. 
Proof. First of all let us assume that $H_{*}^{1}(E) \neq 0$ and $H_{*}^{5}(E) \neq 0$. We can consider a minimal monad for $E$,

$$
0 \rightarrow A \stackrel{\alpha}{\rightarrow} B \stackrel{\beta}{\rightarrow} C \rightarrow 0 .
$$

$B$ satisfies all the hypothesis of [1] Theorem 2.4 so it is a direct sum of bundles $S, S^{\vee}, Q$ and $\mathcal{O}_{G}$ with some twist.

Moreover $B$ must satisfy the conditions $H_{*}^{1}\left(\wedge^{2} B\right) \neq 0$ and $H_{*}^{1}\left(\wedge^{2} B^{\vee}\right) \neq 0$. Since $\wedge^{2} S^{\vee}, \wedge^{2} S$ and $\wedge^{2} Q$ are all ACM bundles and the only non-zero $H^{1}$ cohomology of the tensor product between universal bundles is $h^{1}\left(Q \otimes S^{\vee}\right)=1, B$ must have at least a copy of $Q, S$ and $S^{\vee}$. Assume that more than one copy of $S^{\vee}$ or more than one copy of $S$ appears in $B$. Then in the bundle $\wedge^{2} B$ or in the bundle $\wedge^{2} B^{\vee}$, it appears $\left(S^{\vee} \otimes S^{\vee}\right)(t)$ and, since

$$
H_{*}^{2}\left(S^{\vee} \otimes S^{\vee}\right) \neq 0
$$

the condition

$$
H_{*}^{2}\left(\wedge^{2} B\right)=H_{*}^{2}\left(\wedge^{2} B^{\vee}\right)=0
$$

in Remark 2.2, fails to be satisfied.

We can conclude that $B$ has to be of the form

$$
\left(\bigoplus_{i=1}^{h} \mathcal{O}\left(a_{i}\right)\right) \oplus\left(\bigoplus_{j=1}^{k} Q\left(b_{j}\right)\right) \oplus(S(c)) \oplus\left(S^{\vee}(d)\right),
$$

with $h \geq 0$ and $k \geq 1$.

Let us notice furthermore that $\operatorname{rank}(B)=h+2 k+6$ and $H_{*}^{1}\left(\wedge^{2} B\right) \cong H_{*}^{1}\left(\left(\bigoplus_{j=1}^{k} Q\right) \otimes S^{\vee}\right)$ has $k$ generators. Since $\operatorname{rank}(C)=h+2 k+6-\operatorname{rank}(E)-\operatorname{rank}(A)$, we have

$$
\beta_{0}\left(H_{*}^{0}\left(S_{2} C\right)\right) \geq \beta_{0}\left(H_{*}^{0}(C)\right)=h+2 k+6-\operatorname{rank}(E)-\operatorname{rank}(A) \geq h+2 k+3-\operatorname{rank}(A) .
$$

So $k=\beta_{0}\left(H_{*}^{1}\left(\wedge^{2} B\right)\right) \geq \beta_{0}\left(H_{*}^{0}\left(S_{2} C\right)\right) \geq h+2 k+4+\operatorname{rank}(A)$ which implies $\operatorname{rank}(A) \geq h+k+3$. Moreover $H_{*}^{1}\left(\wedge^{2} B^{\vee}\right) \cong H_{*}^{1}\left(\left(\bigoplus_{j=1}^{k} Q\right) \otimes S\right)$ has $k$ generators. So $k=\beta_{0}\left(H_{*}^{1}\left(\wedge^{2} B\right)\right) \geq$ $\beta_{0}\left(H_{*}^{0}\left(S_{2} A\right)\right) \geq \operatorname{rank}(A) \geq h+k+3$ which is impossible.

Let us assume now that $H_{*}^{1}(E) \neq 0$ and $H_{*}^{5}(E)=0$ (hence $\operatorname{rank}(E)=3$ ). By using the above argument we see that, since $H_{*}^{1}\left(\wedge^{2} B\right) \neq 0$, at least one copy of $S^{\vee}$ must appear in $B$. Moreover, since $H_{*}^{2}\left(\wedge^{2} B\right)=0$, it is no possible to have more than one copy of $S^{\vee}$ We can conclude that $B$ has to be of the form

$$
\left(\bigoplus_{i=1}^{h} \mathcal{O}\left(a_{i}\right)\right) \oplus\left(\bigoplus_{j=1}^{k} Q\left(b_{j}\right)\right) \oplus\left(\left(\bigoplus_{l=1}^{s} S\left(c_{l}\right)\right) \oplus\left(S^{\vee}(d)\right),\right.
$$

with $h, s \geq 0$ and $k \geq 1$.

Let us notice furthermore that $\operatorname{rank}(B)=h+2 k+3 s+3$ and $H_{*}^{1}\left(\wedge^{2} B\right) \cong H_{*}^{1}\left(\left(\bigoplus_{j=1}^{k} Q\right) \otimes\right.$ $\left.S^{\vee}\right)$ has $k$ generators. Since $\operatorname{rank}(C)=h+2 k+3 s$, we have

$$
\beta_{0}\left(H_{*}^{0}\left(S_{2} C\right)\right) \geq \beta_{0}\left(H_{*}^{0}(C)\right)=h+2 k+3 s .
$$

So $k=\beta_{0}\left(H_{*}^{1}\left(\wedge^{2} B\right)\right) \geq \beta_{0}\left(H_{*}^{0}\left(S_{2} C\right)\right) \geq h+2 k+3 s$, which it is impossible.

A symmetric argument show that there are no minimal monads in the case $H_{*}^{1}(E)=0$ and $H_{*}^{5}(E) \neq 0$.

We proved that the every rank $r(r \leq 3)$ bundle without inner cohomology must have $H_{*}^{1}(E)=H_{*}^{5}(E)=0$. Then by [1] Theorem 2.4 they are the claimed. 


\section{References}

[1] E. Arrondo And B. Graña, Vector bundles on $G(1,4)$ without intermediate cohomology, 1999, J. of Algebra 214, 128-142.

[2] E. Arrondo and F. Malaspina, Cohomological Characterization of Vector Bundles on Grassmannians of Lines, 2009, preprint arXiv:0902.2897.

[3] E. Ballico And F. Malaspina, Qregularity and an Extension of the Evans-Griffiths Criterion to Vector Bundles on Quadrics, J. Pure Appl Algebra 213 (2009), 194-202.

[4] L. Chinntini, P. Valabrega, Subcanonical curves and complete intesections in the projective 3-space, 1984, Ann. Mat. Pura Appl. 138, 309-330.

[5] J. V. Chipalkatti, A generalization of Castelnuovo regularity to Grassman varieties, Manuscripta Math. 102 (2000), no. 4, 447-464.

[6] L. Costa And R. M. Miró-Roig, m-blocks collections and Castelnuovo-Mumford regularity in multiprojective spaces, Nagoya Math. J. 186 (2007), 119-155.

[7] G. Horrocks, Vector bundles on the punctured spectrum of a ring, Proc. London Math. Soc. (3) 14 (1964), 689-713.

[8] N.Mohan Kumar, C. Peterson and A.P. RaO, Monads on projective spaces, 2003, Manuscripta Math. 112, 183-189.

[9] F. Malaspina, Monads and Vector Bundles on Quadrics, Adv. Geom. Vol. 9, issue 1, 2009, 137-152.

[10] F. Malaspina, Monads and rank three vector bundles on quadrics, Ann. Mat. Pura Appl. (2009) 188: 455-465.

[11] D. Mumford, Lectures on curves on an algebraic surface, Princeton University Press, Princeton, N.J., 1966.

[12] G. Ottaviani, Criteres de scindage pour les fibres vectoriel sur les grassmanniennes et les quadriques, 1987, C. R. Acad. Sci. Paris, 305, 257-260.

[13] G. Ottaviani, Some extension of Horrocks criterion to vector bundles on Grassmannians and quadrics, 1989, Annali Mat. Pura Appl. (IV) 155, 317-341. 